\title{
EXOTIC COHOMOLOGY AND INDEX THEORY
}

\author{
JOHN ROE
}

\section{INTRODUCTION}

The main result of this paper is a version of the Atiyah-Singer index theorem for Dirac-type operators on (noncompact) complete Riemannian manifolds. The statement of the theorem involves a novel "cohomology" theory for such manifolds. This theory, called exotic cohomology, depends on the structure at infinity of a space; more precisely, it depends on the way that large bounded sets fit together. For each cohomology class in this theory, we define a "higher index" of a Dirac-type operator, enjoying the stability and vanishing properties of the usual Atiyah-Singer index; these higher indices are analogous to the Novikov higher signatures. Our main theorem will compute these higher indices in terms of standard topological invariants. Applications of this index theorem include a different approach to some of the results of Gromov and Lawson [10] on topological obstructions to positive scalar curvature.

The concept of index that we will use involves the $K$-theory functors $K_{0}$ and $K_{1}$ for operator algebras [3, 14]. Suppose that $B$ is an ideal in a unital algebra $C$, and let $T \in C$ be invertible modulo $B$. (In the classical Atiyah-Singer index theorem, one takes $C$ to be the bounded operators on the $L^{2}$ space of some compact manifold, $B$ the compact operators, and $T$ an elliptic pseudodifferential operator of order zero.) Then $T$ has an "index" in the $K$-theory group $K_{0}(B)$ (in the classical case this is just $\mathbf{Z}$, and one recovers the usual Fredholm index). Now let $M$ be a complete Riemannian manifold, possibly noncompact. In [18] I introduced an algebra $\mathscr{X}(M)$ which is defined as follows: $\mathscr{X}(M)$ consists of all bounded operators $A$ on $L^{2}(M)$ that have a kernel representation

$$
A u(x)=\int k(x, y) u(y) d y,
$$

Received by the editors July 25, 1989 and, in revised form, April 11, 1990.

1980 Mathematics Subject Classification (1985 Revision). Primary 57G12; Secondary $19 \mathrm{~K} 56,55 \mathrm{~N} 35$. 
where the kernel $k(x, y)$ is smooth on $M \times M$ and vanishes whenever the distance $d(x, y)$ is greater than some constant depending only on the operator $A$. One can show, using a variant of the construction above, that a Dirac-type operator $D$ on $M$ has an index in $K_{i}(\mathscr{X}(M))$, where $i=\operatorname{dim}(M) \bmod 2$. The usefulness of this construction depends on how well one understands the groups $K_{i}(\mathscr{Z}(M))$ !

One way to partially understand these groups is to construct homomorphisms $K_{i}(\mathscr{Z}(M)) \rightarrow$ C. Such homomorphisms can be obtained from the cyclic cohomology theory of A. Connes [4]. This theory associates to an algebra such as $\mathscr{X}(M)$ a series of complex vector spaces $H C^{q}(\mathscr{X}(M))$, and there is a natural pairing (the Chern pairing)

$$
H C^{q}(\mathscr{X}(M)) \otimes K_{[q]}(\mathscr{X}(M)) \rightarrow \mathbf{C},
$$

where $[q]$ denotes $q$ mod 2 . Thus, we look for cyclic cohomology classes for $\mathscr{X}(M)$.

Such cyclic cohomology classes can be obtained from exotic cohomology. The exotic cohomology groups $H X^{q}(M)$, which depend on the geometry of $M$, are constructed in such a way that there is a "character" map $\chi: H X^{q}(M) \rightarrow H C^{q}(\mathscr{X}(M))$. The concept of exotic cohomology is motivated by the construction of [18]. There it was shown that a partition of the manifold $M$, that is a compact hypersurface whose complement is disconnected, determines in a natural way a 1-dimensional cyclic class for $\mathscr{X}(M)$. It can be shown that $H X^{1}(M)$ is generated in a natural way by such partitions, so one can think of exotic cohomology as generalizing the partition construction to higher codimensions. There is, however, a significant difference: in contrast to $H X^{1}$, the higher exotic cohomology groups depend on the metric, and not just on the topological, structure of $M$. For example, one can show that $H X^{2}\left(\mathbf{R}^{2}\right)$ is 1-dimensional, but if $M$ denotes the manifold, diffeomorphic to $\mathbf{R}^{2}$, obtained by smoothly capping off a semi-infinite cylinder, then $H X^{*}(M)$ is trivial. Exotic cohomology is an essentially noncompact theory: every compact manifold has the same exotic cohomology as a point.

Given therefore an operator $D$ of Dirac type on $M$ and an exotic class $\varphi$ in $H X^{*}(M)$ of appropriate parity, one can obtain a "real-valued index" $\langle$ Ind $D, \chi[\varphi]\rangle$. Our main theorem will then compute this index in topological terms, using the standard index class $\operatorname{ch}(\sigma(D)) \smile \operatorname{td}(T M) \in H^{*}(M)$ associated to $D[1,2]$ and 
a topological character map $c: H X^{*}(M) \rightarrow H_{c}^{*}(M)$. At the end of this paper we will give an example showing how this result can be applied in conjunction with Bochner vanishing arguments to obtain results about the impossibility of positive scalar curvature metrics on certain manifolds. Further examples will appear in [15].

\section{DEFINITION, BASIC PROPERTIES}

Let $M$ be a complete metric space in which closed bounded sets are compact. Let $C X^{q}(M)$ denote the space of all continuous, antisymmetric functions $\varphi: M^{q+1} \rightarrow \mathbf{R}$ which satisfy the following support condition: Let $\Delta=\{(x, \ldots, x): x \in M\}$ be the multidiagonal in $M^{q+1}$, and for $R>0$ let $\operatorname{Pen}(\Delta ; R)$ denote the $R$-neighborhood of $\Delta$; we require that for any $R>0$, $\operatorname{Supp}(\varphi) \cap \operatorname{Pen}(\Delta ; R)$ be relatively compact in $M^{q+1}$. The vector spaces $C X^{q}(M)$ then form a cochain complex under the usual boundary operator $\partial$ of Alexander-Spanier cohomology theory [21], and we define the exotic cohomology $H X^{*}(M)$ to be the cohomology of this complex.

The idea of relating Alexander-Spanier cohomology to index theory was introduced by Connes and Moscovici [5, 6]. Exotic cohomology leads to a "delocalized" version of their index theorem.

For example, let $M=\mathbf{R}^{n}$ and define $\varphi$ as follows: Let $\alpha$ be a compactly supported $n$-form on $M$ with $\int \alpha=1$, and for $\left(x_{0}, \ldots, x_{n}\right) \in M^{n+1}$ let $\Lambda\left(x_{0}, \ldots, x_{n}\right)$ denote the oriented affine $n$-simplex in $M$ with vertices $x_{0}, \ldots, x_{n}$; set

$$
\varphi\left(x_{0}, \ldots, x_{n}\right)=\int_{G k\left(x_{0}, \ldots, x_{n}\right)} \alpha .
$$

It is then easily checked that $\varphi$ is an exotic $n$-cocycle; and it can be proved that $[\varphi]$ in fact generates $H X^{*}(M)$ as a real vector space.

The main computational tool for $H X^{*}$ is a variation of the Čech approach to cohomology: Roughly speaking, whereas to compute ordinary cohomology one considers the Čech theory of successively finer coverings and passes to a direct limit, to compute exotic cohomology one considers the Čech theory of successively coarser coverings and passes to an inverse limit. In this way one can compute exotic cohomology in many examples, and one can prove, for instance, that if the Hausdorff distance $d_{H}(M, N)$ (in the sense of Gromov [9]) is finite, then $H X^{*}(M) \cong H X^{*}(N)$. 
Exotic cohomology is functorial under proper maps $f: M \rightarrow N$ that satisfy the condition: For all $R>0$ there exists $S>0$ such that $d\left(x, x^{\prime}\right)<R \Rightarrow d\left(f(x), f\left(x^{\prime}\right)\right)<S$. For complete Riemannian manifolds this property is implied by uniformly continuity.

\section{ChaRACTER MAPS AND INDEX THEORY}

There is a homomorphism $c: H X^{*}(M) \rightarrow H_{c}^{*}(M)$. If we think of the right-hand side of Alexander-Spanier cohomology with compact supports, then $c[\varphi]$ is just the equivalence class of $\varphi$ modulo locally zero cochains.

Let $M$ be a complete Riemannian manifold, and let $\mathscr{X}(M)$ denote the algebra introduced in [18]. Let $H C^{*}(\mathscr{X})$ denote Connes cyclic cohomology of $\mathscr{X}$ [4]. There is a Connes character map $\chi: H X^{*}(M) \rightarrow H C^{*}(\mathscr{X})$ defined as follows $[5,6]:$ For an exotic $q$-cocycle $\varphi$, define

$$
\begin{aligned}
& \chi[\varphi]\left(A_{0}, \ldots, A_{q}\right) \\
& =\int \cdots \int k_{0}\left(x_{0}, x_{1}\right) k_{1}\left(x_{1}, x_{2}\right) \\
& \quad \cdots k_{q}\left(x_{q}, x_{0}\right) \varphi\left(x_{0}, \ldots, x_{q}\right) d x_{0} \cdots d x_{q},
\end{aligned}
$$

where the operators $A_{i} \in \mathscr{X}$ have kernels $k_{i}$. Although the integration is taken over the noncompact space $M \times \cdots \times M$, the support conditions on $\varphi$ and on the $k_{i}$ interact to show that the integral must converge - the range of integration is contained in $\operatorname{Supp}(\varphi)$ and must also be contained in some bounded neighborhood of the diagonal.

Now let $D$ be an operator of Dirac type on the complete Riemannian manifold $M$. Then $[16,17,18] D$ has an index $\operatorname{Ind}(D)$ $\in K_{i}(\mathscr{X}(M))$, where $i=\operatorname{dim}(M) \bmod 2$. (Strictly speaking, when $i$ is odd we take $K_{-1}$ rather than $K_{1}$.) Now Connes [4] defines a pairing between $K$-theory and cyclic cohomology, which we can use to pair $\operatorname{Ind}(D)$ with the Connes character of an exotic cohomology class. Our main result is then:

Theorem. Let $[\varphi] \in H X^{q}(M)$. Then

$$
\langle\text { Ind } D, \chi[\varphi]\rangle=a_{q}\langle c[\varphi] \smile \operatorname{ch}(\sigma(D)) \smile \operatorname{td}(M),[M]\rangle,
$$

where the nonzero constant $a_{q}$ depends only on $q$.

For $q=1$ this index theorem incorporates the results of [18]. For $q=0$ it is the ordinary Atiyah-Singer index theorem [2]. The proof uses some of the remarkable results of Connes and Moscovici 
[6], specifically their application to cyclic cohomology of Getzler's [8] asymptotic pseudodifferential calculus and the resulting localized index theorem.

\section{Corona SPACE}

Let $M$ be a complete Riemannian manifold. In [12], Higson introduced a compactification $\bar{M}$ of $M$, defined as the maximal ideal space of the commutative $C^{*}$-algebra generated by smooth bounded functions $f$ on $M$ such that $\nabla f \rightarrow 0$ at infinity. Higson's compactification is related to, but is somewhat larger than, the Freudenthal compactification [7]. Let $\nu M=\bar{M} \backslash M$ be the "corona" of $\bar{M}$. Higson shows that there is a natural map $K_{i}(\overline{\mathscr{X}})$ $\rightarrow K_{i \pm 1}(\nu M)$, where the left-hand side is the $K$-theory of the $C^{*}$-algebraic closure $\overline{\mathscr{X}}$ of $\mathscr{X}$ and the right-hand side is the $K$ homology of the space $\nu M$. Dually, I prove that there is a "transgressed Chern character" Tch : $K^{1}(\nu M) \rightarrow H X^{\text {even }}(M), K^{0}(\nu M)$ $\rightarrow H X^{\text {odd }}(M)$. These constructions are compatible in the sense that if $\operatorname{Ind}(D) \in K_{i}(\mathscr{X}), \psi \in K^{i \pm 1}(\nu M)$, then

$$
\langle b(\operatorname{Ind}(D)), \psi\rangle=\langle\operatorname{Ind}(D), \chi[\operatorname{Tch}(\psi)]\rangle,
$$

where $b: K_{i}(\mathscr{X}) \rightarrow K_{i}(\overline{\mathscr{X}}) \rightarrow K_{i \pm 1}(\nu M)$ is Higson's map. The relevance of this is that if $D$ has an inverse bounded on $L^{2}(M)$, then one can show that $\operatorname{Ind}(D)$ vanishes in $K_{*}(\overline{\mathscr{X}})$, although not necessarily in $K_{*}(\mathscr{X})$. Therefore, if $D$ has a bounded inverse, $\langle\operatorname{Ind}(D), \chi[\varphi]\rangle=0$ for any $\varphi \in \operatorname{Im}(\operatorname{Tch} \otimes \mathbf{R})$.

Example. Let us use these techniques to reprove the famous result of Schoen and Yau [19, 20], and of Gromov and Lawson [10, 11] that the torus $\mathbf{T}^{n}$ has no metric of positive scalar curvature. Let $M=\mathbf{R}^{n}, \varphi \in H X^{n}(M)$ the class defined in $\S 1$, and $D$ the classical Dirac operator. Suppose the torus admitted a metric of positive scalar curvature. This would then lift to a metric on $M$, uniformly equivalent to the standard one-and hence having the same exotic cohomology. The index theorem of $\S 3$ shows that $\langle\operatorname{Ind}(D), \chi[\varphi]\rangle \neq 0$; but it can be shown that $[\varphi] \in \operatorname{Im}(\operatorname{Tch} \otimes \mathbf{R})$, and $D$ has a bounded inverse by the Lichnerowicz-Weitzenbock formula [13], so by the results of $\S 4,\langle\operatorname{Ind}(D), \chi[\varphi]\rangle=0$. This contradiction establishes the result.

Note. These results were presented at a conference held at Boulder, Colorado, in April 1989. I am grateful to all the participants in the conference, and especially to Nigel Higson and Steven Hurder, 
for their helpful questions and remarks. Full details will appear elsewhere [15].

\section{REFERENCES}

1. M. F. Atiyah, R. Bott and V. K. Patodi, On the heat equation and the index theorem, Invent. Math. 19 (1973), 279-330.

2. M. F. Atiyah and I. M. Singer, The index of elliptic operators III. Ann. of Math. (2) 87 (1968), 546-604.

3. B. Blackadar, K-theory for operator algebras, Mathematical Sciences Research Institute Publications, Springer-Verlag, New York, 1986.

4. A. Connes, Non-commutative differential geometry, Publ. Math. l'Institut des Hautes Études Scientifiques 62 (1985), 141-144.

5. A. Connes and H. Moscovici, Conjecture de Novikov et groupes hyperboliques, C. R. Acad. Sci. Paris Ser. A 307 (1988), 475-479.

6. Cyclic cohomology, the Novikov conjecture, and hyperbolic groups, Topology (1990) (to appear).

7. H. Freudenthal, Neuaufbau der endentheorie, Ann. of Math. 43 (1942), 261-279.

8. E. Getzler, Pseudodifferential operators on supermanifolds and the AtiyahSinger index theorem, Comm. Math. Phys. 92 (1983), 163-178.

9. M. Gromov, Structures métriques pour les variétés Riemanniennes, Editions CEDIC, Paris, 1981.

10. M. Gromov and H. B. Lawson, Positive scalar curvature and the Dirac operator, Publ. Math. l'Institut des Hautes Études Scientifiques 58 (1983), 83-196.

11. $\ldots$ Spin and scalar curvature in the presence of a fundamental group, Ann. of Math. 111 (1980), 209-230.

12. N. Higson, On the relative $K$-homology theory of Baum and Douglas, preprint, 1989.

13. A. Lichnerowicz, Spineurs harmoniques, C. R. Acad. Sci. Paris Ser. A 257 (1963), 7-9.

14. J. W. Milnor, Introduction to algebraic $K$-theory, Princeton University Press, 1971.

15. J. Roe, Exotic cohomology and index theory on complete Riemannian manifolds, preprint, 1990.

16. _ An index theorem on open manifolds I, J. Differential Geom. 27 (1988), 87-113.

17. __ Operator algebras and index theory on non-compact manifolds, Index Theorey of Elliptic Operators, Foliations, and Operator Algebras (C. Miller, J. Kaminker, and C. Schocher, eds.), Amer. Math. Soc., Providence, R.I., 1988, pp. 229-249.

18. _ Partitioning non-compact manifolds and the dual Toeplitz problem, Operator Algebras and Applications (D. Evans and M. Takesaki, eds.), Cambridge Univ. Press, Cambridge, 1989, pp. 187-228.

19. R. Schoen and S. T. Yau, Existence of incompressible minimal surfaces and the topology of three dimensional manifolds of non-negative scalar curvature, Ann. of Math. 110 (1979), 127-142. 
20. _ On the structure of manifolds with positive scalar curvature, Manuscripta Math. 28 (1979), 159-183.

21. E. Spanier, Algebraic topology, McGraw-Hill, 1966.

Department of Mathematics, Jesus College, University of Oxford, OXFORD OX1 30W, ENGLAND 
